\title{
Diagnostic Significance of Computed Tomography in Unilateral Exophthalmos
}

\author{
Hayao Shiga, Akira Yagishita, Taketo Tsutsui, \\ Shimakichi SuzuKI, Kiyoshi IchIKIZaKI, ${ }^{*}$ Kyojiro KImURA, ${ }^{* *}$ \\ Yoichi INOUE*** and Kunihiko ITO**** \\ Departments of Diagnostic Radiology, Neurosurgery* and \\ Ophthalmology,** Keio University School of Medicine, \\ Olympia Clinic ${ }^{* * *}$ and Ito Hospital ${ }^{* * * *}$
}

\begin{abstract}
Summary
CT is very useful for differential diagnosis of unilateral exophthalmos. Lesions extending from the cranial cavity or the paranasal sinus into the orbit can be diagnosed easily by this method. About two thirds of patients with dysthyroid ophthalmopathy have enlargement of the extraocular muscles, and the remaining one third have increase in fatty tissues. Inflammation in the orbit and malignant tumor usually show a diffuse high density which cannot be differentiated from normal intraorbital structures. Benign intraorbital tumor has a localized high density mass and shows definite demarcation. Histological diagnosis frequently cannot be made from absorption density value and contrast enhancement. Therefore, the patient's age, clinical symptoms, site of lesion and angiographic findings should be considered.
\end{abstract}

Key words: Computed tomography, orbital lesion, unilateral exophthalmos

\section{Introduction}

Since the development of CT, the effectiveness of this method to diagnose intracranial disease has been demonstrated by many scholars. Compared to usual radiological examinations, it has become evident that $\mathrm{CT}$ is quite useful in diagnosing orbital lesions. ${ }^{1,2.7-9.11-15}$ )

The common orbital lesions with unilateral exophthalmos are dysthyroid ophthalmopathy and orbital tumor, but also there are many other lesions. The purpose of this paper is to determine whether or not $\mathrm{CT}$ can be used to differentiate various kinds of lesions that show unilateral exophthalmos.

\section{Materials and Methods}

CT findings in 58 patients with unilateral exophthalmos were analyzed for a one year period from October 1976 to September 1977. All the patients were examined with EMIScanner CT-1000 using $8 \mathrm{~mm}$. collimation. The scanning plane was parallel to Reid's base line, and each slice was overlapped slightly.

Twenty five patients had intraorbital spaceoccupying lesions, 17 had dysthyroid ophthalmopathy, 5 had lesion extending from the cranial cavity to the orbit, 4 had lesion extending from the paranasal sinus to the orbit, and in 7 the cause of the lesion was unknown (Table 1).

Of the patients with intraorbital spaceoccupying lesions, 15 had tumor and 10 had inflammation. Fifteen cases of tumor included 5 lacrimal tumors, 4 hemangiomas, 1 optic

Table 1 Causes of unilateral exophthaimos

\begin{tabular}{lrr}
\hline Intraorbital space-occupying lesions & 25 & \\
$\quad$ Tumor & & 15 \\
$\quad$ Inflammation & 17 \\
Dysthyroid ophthalmopathy & 17 \\
Intracranial lesions & 5 \\
Lesions arising in the sinuses & 4 \\
Unknown & 7 \\
& -58 cases
\end{tabular}


glioma, 1 epidermoid, 1 malignant lymphoma, and 3 unknown tumors. Ten cases of inflammation included 2 abscesses, and 8 pseudotumors (Table 2).

Twelve females and five males had dysthyroid ophthalmopathy (Table 3).

Of five patients having intracranial lesions, 3 had meningioma, 1 had fibrous dysplasia, and 1 had neurinoma. Of 4 patients having lesions in the paranasal sinus, 3 had mucocele and 1 had carcinoma (Table 4).

Table 2 Classification of intraorbital tumors and inflammations

\section{Tumors}

Lacrimal tumor

Hemangioma

Optic glioma

Epidermoid

Malignant lymphoma

Unknown

Inflammations

Abscess

Pseudotumor

\begin{tabular}{l}
2 \\
8 \\
\hline 10 cases
\end{tabular}

Table 3 Sex, side of exophthalmos, and muscle involvement in dysthyroid ophthalmopathy

\section{Sex Male \\ Female}

Side of exophthalmos

Muscle enlargement

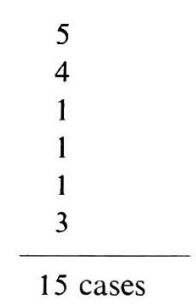

Right $\quad 6$

Bilateral 5

Table 4 Lesions extending from the cranial cavity and the paranasal sinus

Intracranial lesions

Meningioma

Neurinoma

Fibrous dysplasia

Unilateral 5

Negative 7

17 cases

cases

Lesions arising in the paranasal sinus

Mucocele

Carcinoma

\begin{tabular}{l}
3 \\
1 \\
\hline 4 cases
\end{tabular}

Mucocele in the paranasal sinus, which extended to the orbit, could be diagnosed more easily by $\mathrm{CT}$ than plain radiography or tomography. Increased density in the paranasal sinus and the presence of a thin and expanded bony wall around it were revealed (Fig. 1)

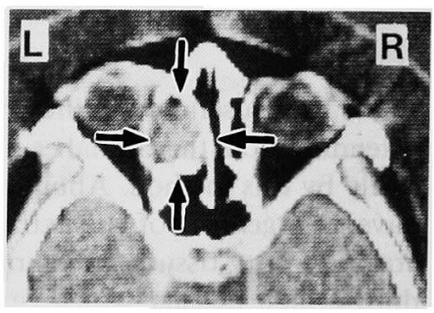

a

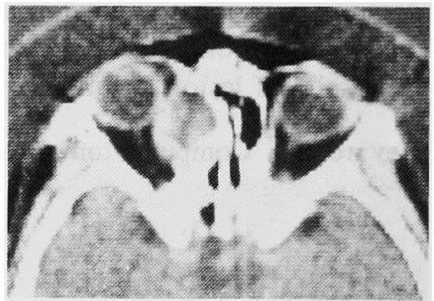

b

Fig. 134 y. male; left ethmoid mucocele $(\rightarrow)$. a: plain $\mathrm{CT}$; b: contrast CT.

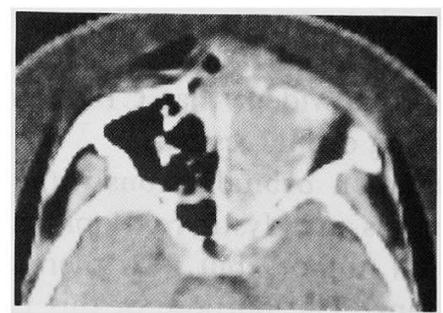

CT findings were studied with special reference to the site of lesion and bony change.

\section{Results}

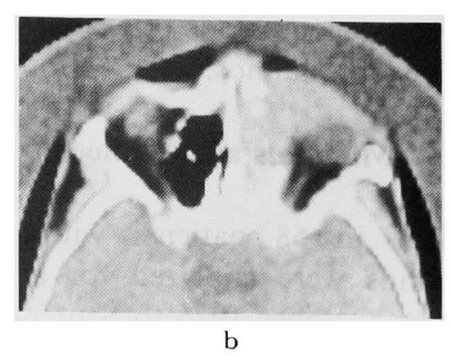

Fig. 256 y. male; right maxillary carcinoma. 
Diffuse high density, which could not be differentiated from the normal intraorbital structures, was present in case of developed carcinoma from the paranasal sinus, in addition to the presence of diffuse density in the
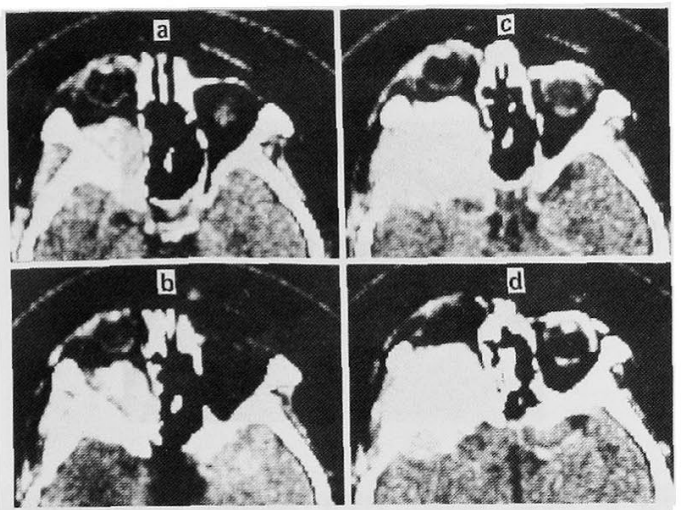

Fig. 359 y. male; left shenoidal meningioma. a, b: plain CT; c, d: contrast CT.
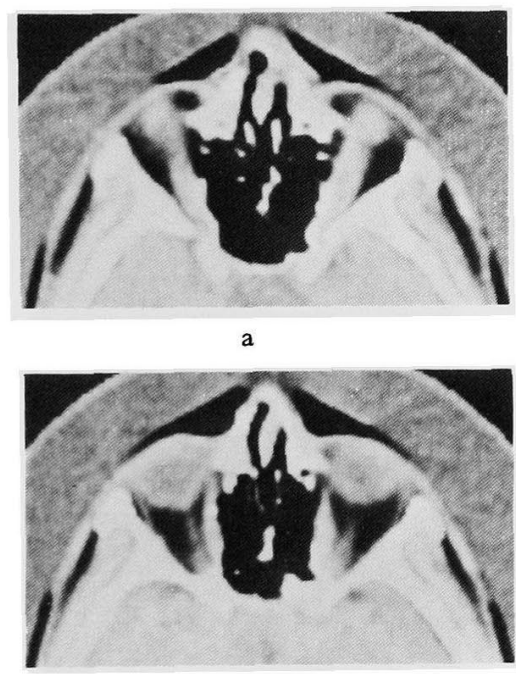

b

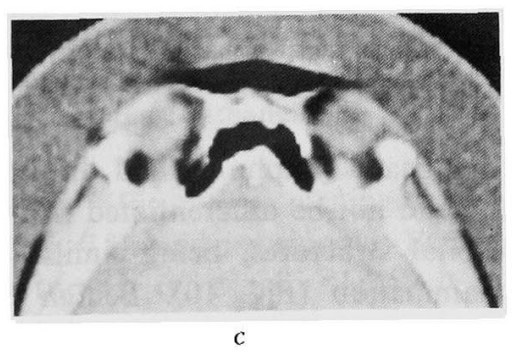

Fig. 430 y. male; dysthyroid ophthalmopathy; right exophthalmos. There is marked bilateral enlargement of the inferior, medial rectus muscles and superior group of muscles (superior rectus and levator palpebral superioris). paranasal sinus and destruction of bones (Fig. 2). In cases of intracranial meningioma, a mass showing marked contrast enhancement was present both in the cranium and in the orbit, in addition to a thickened bone shadow (Fig. 3). In case of fibrous dysplasia, although bones had thickened, a soft tissue mass was not present. In case of neurinoma, a markedly enhanced mass with contrast media was clearly identified but bones had not thickened.

Dysthyroid ophthalmopathy is usually accompanied by bilateral exophthalmos, but 17 cases showed unilateral exophthalmos among 167 patients examined with CT. It is accepted that CT findings in cases of dysthyroid ophthalmopathy are characterized by enlarged extraocular muscles. ${ }^{5.10 .14)}$ In this study, 5 patients revealed enlargement of bilateral extraocular muscles (Fig. 4), and 5 showed enlargement of unilateral extraocular muscles (Fig. 5). In the remaining 7 patients, the
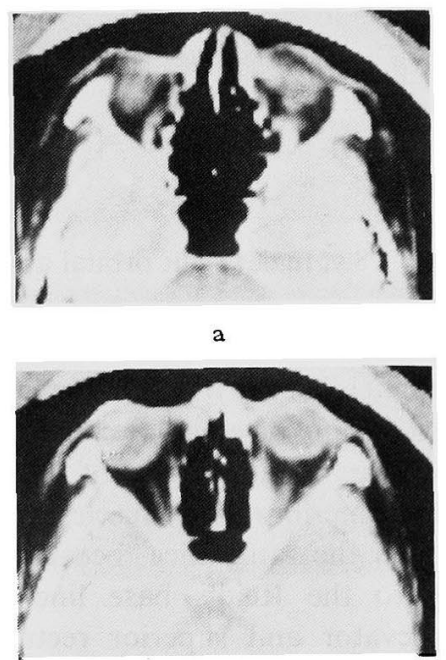

b

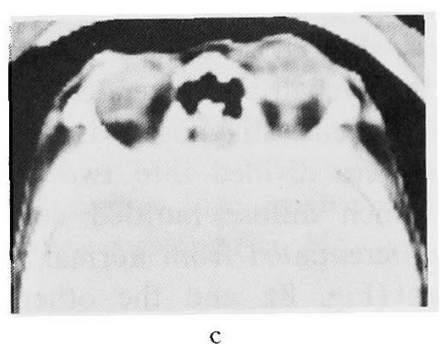

Fig. 551 y. female; dysthyroid ophthalmopathy; right exophthalmos. There is moderate enlargement of the right inferior, medial rectus muscles and superior group of muscles. 


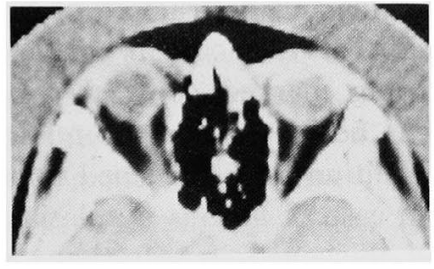

a

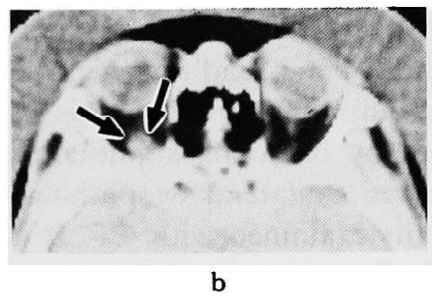

Fig. $648 \mathrm{y}$. male; dysthyroid ophthalmopathy, left exophthalmos. Soft tissue mass of the left orbital apex shows the enlarged superior group of muscles $(\rightarrow)$.

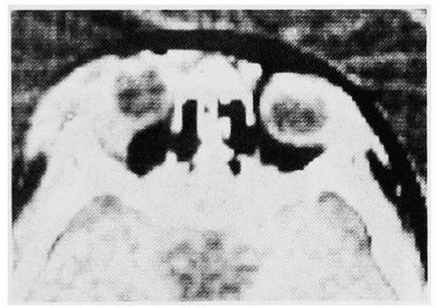

Fig. 78 y. male; right orbital abscess.

extraocular muscles had not enlarged. On slices parallel to Reid's base line, the optic nerve and inferior rectus muscle could be clearly identified. However, the whole length of the superior levator and superior rectus muscles could not be identified on the same slice, because they ran obliquely to the Reid's base line. Enlarged superior levator and superior rectus muscles were sometimes observed as a soft tissue mass of the orbital apex (Fig. 6).

Abscess showed a diffuse mottled density around the eyeball and extended to the eyelid (Fig. 7). Patients diagnosed as orbital pseudotumor were divided into two groups: the one in which diffuse mottled density could not be differentiated from normal intraorbital structures (Fig. 8), and the other in which diffuse mottled density was comparatively localized and was accompanied by thickening of the extraocular muscles (Fig. 9).

All our cases of orbital tumors, except malignant lymphoma, were benign. Patients

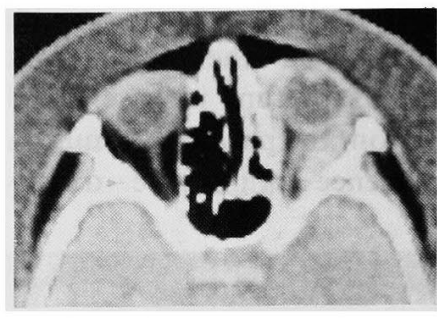

a

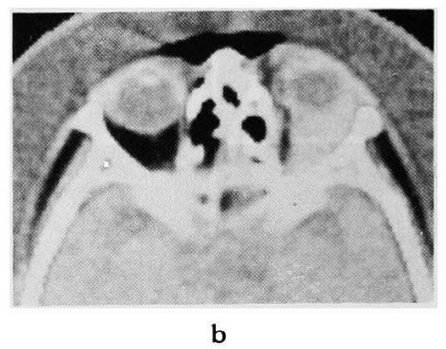

Fig. 865 y. male; right orbital pseudotumor.

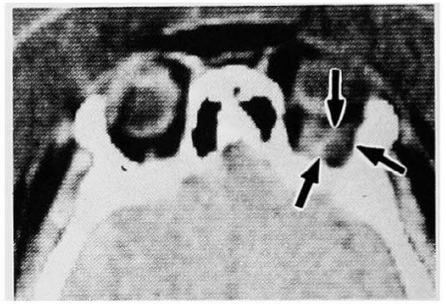

a

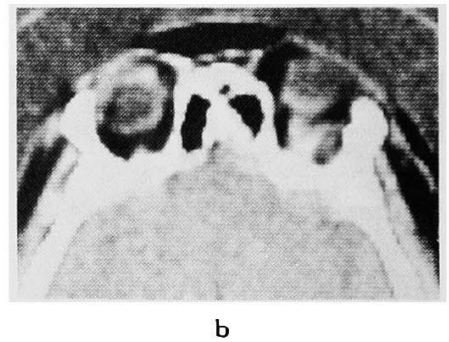

Fig. 936 y. male; a: plain CT; b: contrast CT; right orbital pseudotumor. There is enlargement of right lateral rectus muscle with other abnormal density $(\rightarrow)$.

with malignant lymphoma had diffuse density which could not be differentiated from normal intraorbital structures, being similar to cases of inflammation (Fig. 10). Benign tumor is usually observed as a well-defined high density mass. However, in only one infant with capillary hemangioma, a diffuse density was observed (Fig. 11). CT makes it possible to determine whether a mass is intraconic or extraconic in location. Intraconic tumors include cavernous 


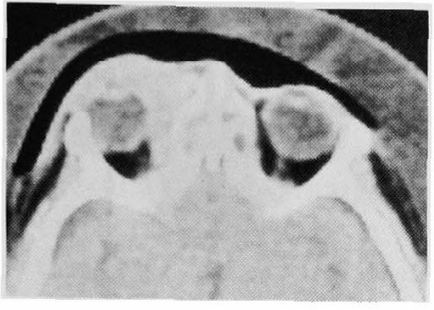

a

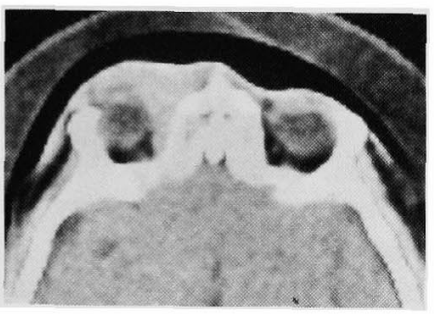

b

Fig. 1029 y. male; a: plain CT; b: contrast CT; left orbital malignant lymphoma.

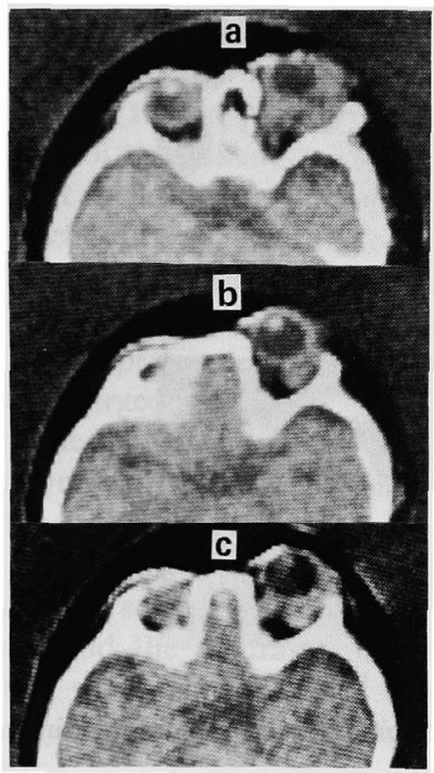

Fig. 113 y. female; a, b: plain CT; c: contrast $\mathrm{CT}$; right capillary hemangioma.

hemangioma (Fig. 12), meningioma, optic glioma (Fig. 13), and neurinoma. Because the lacrimal tumor is usually located in the superolateral quadrant of the anterior orbit, and because it can be easily diagnosed clinically, CT is useful in evaluating whether a tumor is benign or malignant. Benign lacrimal tumors always show definite demarcation (Fig. 14), while in cases of malignant tumors some parts
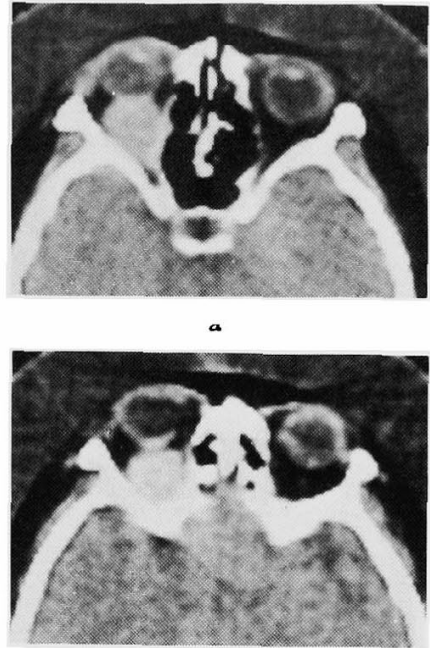

b

Fig. 1248 y. female; a: plain $\mathrm{CT}$; b: contrast CT; left cavernous hemangioma.
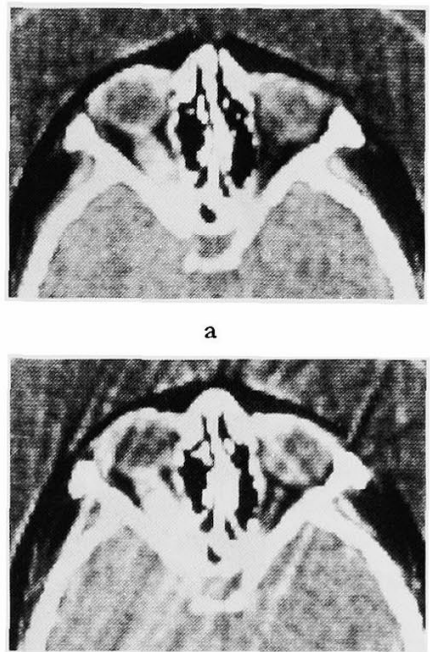

Fig. 1316 y. female; a: plain CT; b: contrast CT; left optic glioma.

of the mass show indefinite demarcation (Fig. 15). In case of epidermoid, CT findings show a ring-like high density corresponding to the capsule with central low density, and accompanied by bone erosion (Fig. 16).

\section{Discussion}

Intraorbital lesions almost always show an abnormally high density because there is fatty tissue in the orbit, which act as a negative 


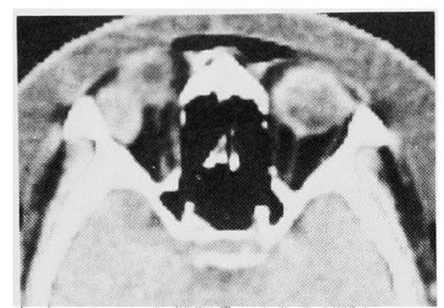

a

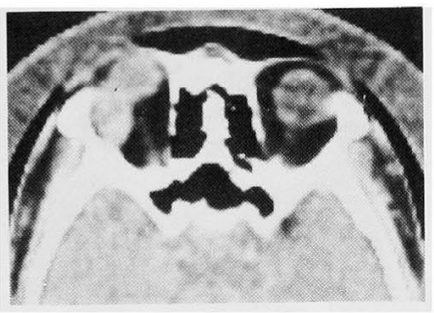

b

Fig. 1433 y. male; a: plain CT; b: contrast CT; left lacrimal mixed tumor.

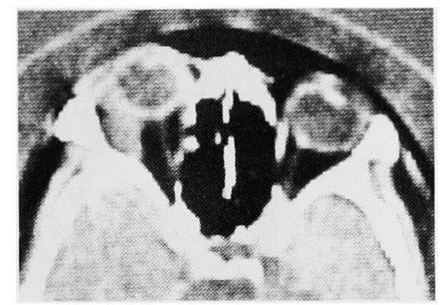

a

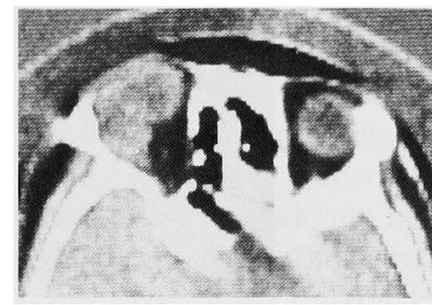

b

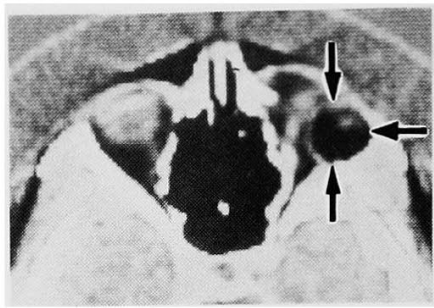

a

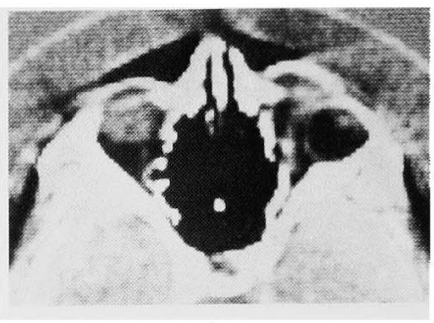

b

Fig. 1636 y. male; a: plain CT; b: contrast CT; right intraorbital epidermoid $(\rightarrow)$.

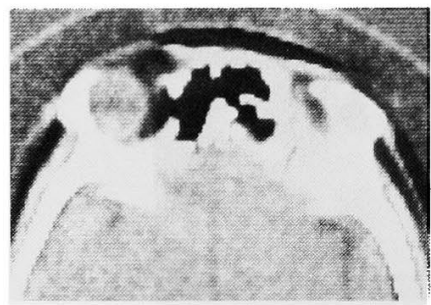

c

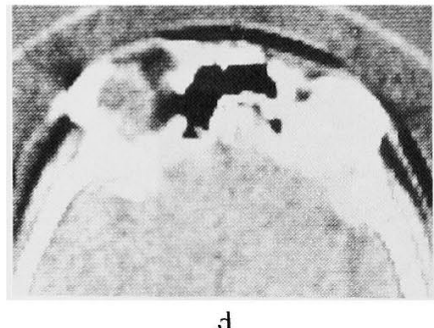

$d$

Fig. 1526 y. male; left lacrimal adenoid cystic carcinoma. Slice b shows indefinite margin adjacent to the globe.

contrast media. The absorption density value of the lesion, the degree of contrast enhancement, shape and extension of the lesion, deviation and deformity of the intraorbital structures, and changes in the orbital wall are considered to be important diagnostic criteria of orbital lesions. However, in cases of orbital lesions, absorption density values are almost the same, and the contrast enhancement contributes very little to the differential diagnosis. ${ }^{11,15}$ ) Therefore, the most important CT findings of the orbital lesion are the site and extension of the lesion and changes in the orbital bony wall. If the main lesion is located in the cranial cavity or in the paranasal sinus, the intraorbital lesion is diagnosed to be extended from the cranial cavity or the paranasal sinus. In these instances, changes such as destruction of the orbital wall can be observed.

In cases of dysthyroid ophthalmopathy, exophthalmos is usually observed bilaterally. Of the patients studied, about $10 \%$ had 
unilateral exophthalmos. One third of the dysthyroid ophthalmopathy cases accompanied by unilateral exophthalmos showed enlargement of the bilateral extraocular muscles. Another one third had enlargement of the unilateral extraocular muscles. In the remaining one third, no abnormal findings were revealed with the exception of exophthalmos, which seemed to result from increase in fatty tissue. ${ }^{5)}$ Therefore, when unilateral exophthalmos is accompanied only by enlargement of the extraocular muscles or when the patient shows no abnormality on CT, an endocrinological examination should be made. Although past histories of hyperthyroidism existed, many of the patients were in euthyroid or hypothyroid state. ${ }^{10}$ )

Intraorbital inflammation often shows a diffuse high density which cannot be differentiated from normal intraorbital structures. However, it is reported that it also shows a mottled density with an absorption density value of less than EMI number zero, differing from malignant tumor. ${ }^{11)}$ There was a report in which half of the patients with pseudotumor in the orbit had scleral thickening. ${ }^{3)}$ Some cases of pseudotumor had enlargement of the extraocular muscles but could be differentiated from patients with dysthyroid ophthalmopathy because of the presence of abnormal shadow other than enlarged muscles. ${ }^{6}$ )

Most of the tumors showing a localized mass were benign. Cavernous hemangioma was shown to be an intraconic round high density mass. It was reported that the same findings were revealed in some cases of meningioma and neurinoma, and the contrast enhancement of meningioma was most marked. ${ }^{8)}$ However, it is frequently impossible to identify the nature of the lesion by $C T$, so that radiological examination is required for differential diagnosis.

Lacrimal tumor can be diagnosed easily not only from a clinical point of view but from CT findings because of its characteristic site. In cases of malignant lacrimal tumors, part of the border of the tumor is obscure, because it has an infiltrative nature.

\section{Conclusions}

1) Lesions extending from the cranial cavity and paranasal sinus into the orbit could be observed with ease by $\mathrm{CT}$.

2) CT findings of dysthyroid ophthalmopathy were characterized by enlargement of the extraocular muscles but sometimes only showed increase in fatty tissue.

3) Malignant tumor and inflammation showed diffuse high density which could not be differentiated from the normal intraorbital structures.

4) Localized high density mass was present in case of benign tumor.

5) It was frequently impossible to qualitatively diagnose benign tumor from absorption density value and contrast enhancement. Therefore, the patient's age, clinical symptoms, the site of lesion, and angiographic findings should be considered.

\section{References}

1) Ambrose, J. A. E., Lloyd, G. A. S. and Wright, J. E.: A preliminary evaluation of fine matrix computerized axial tomography (Emiscan) in the diagnosis of orbital spaceoccupying lesions. $B r J$ Radiol 47: 747-751, 1974.

2) Bergstrom, K.: Computer tomography of the orbits. Acta Radiol [Suppl] 346: 155-160,1975.

3) Bernardino, M. E., Zimmerman, R. D., Citrin, C. M. and Davis, D. O.: Scleral thickening: A CT sign of orbital pseudotumor. Am J Roentgenol 129: 703-706, 1977.

4) Brismar, J., Davis, K. R., Dallow, R. L. and Brismar, G.: Unilateral endocrine exophthalmos. Diagnostic problems in association with computed tomography. Neuroradiology 12: 21-24, 1976.

5) Enzmann, D., Marshall, W. M., Rosenthal, A. R. and Kriss, J. P.: Computed tomography in Graves' ophthalmopathy. Radiology 118: 615-620, 1976.

6) Enzmann, D., Donaldson, S. S., Marshall, W. H. and Kriss, J. P.: Computed tomography in orbital pseudotumor (idiopathic orbital inflammation). Radiology 120: 597601, 1976.

7) Gawler, J., Sanders, M. D., Bull, J. W. D., du Boulay, G. and Marshall, J.: Computer assisted tomography in orbital disease. $\mathrm{Br} . J$ Ophthalmol 58: 571-587, 1974.

8) Gyldensted, C., Lester, J. and Fledelius, H.: Computed tomography of orbital lesions. A radiological study of 144 cases. Neuroradiology 13: 141-150, 1977.

9) Hilal, S. K. and Trokel, S. L.: Computerized 
tomography of the orbit using thin sections. Semin Roentgenol 12: 137-147, 1977.

10) Inoue, Y., Inoue, T., Ichikizaki, K., Shiga, H. and Yagishita, A.: The role of extraocular muscle in the development of dysthyroid ophthalmopathy. Jpn J Clin Ophthalmol 32: 901-905, 1978.

11) Lloyd, G. A. S. and Ambrose, J. A. E.: An evaluation of C.A.T. in the diagnosis of orbital lesions. pp 154-160, In du Boulay, G. H. and Moseley, I. F. (eds): The First European Seminars on Computerized Axial Tomography in Clinical Practice. Springer-Verlag, Berlin Heiderberg, New York, 1977.

12) Momose, K. J., New, P. F. J., Grove, A. S., Jr. and Scott, W. R.: The use of computed tomography in ophthalmology. Radiology 115: 361-368, 1975.

13) Salvolini, U., Menichelli, F. and Pasquini, U.: Computer assisted tomography in ninety cases of exophthalmos. $J$ Comput Assist Tomogr 1: 81-100, 1977.

14) Shiga, H.: Computed tomography of the orbital lesions. Advances in Neurological Sciences 22: 333-343, 1978.

15) Wende, S., Aulich, A., Nover, A., Lanksch, W., Kazner, E., Steinhoff, H., Meese, W., Lange, S. and Grumme, T.: Computed tomography of orbital lesions. A cooperative study of 210 cases. Neuroradiology 13: 123-134, 1977. 